\title{
Validity of systemic antibiotics and systemic corticosteroid usage for chronic rhinosinusitis as metrics of disease burden*
}

Katie M. Phillips', Marlene M. Speth², Edina T. Shu³, Rehab Talat4, David S. Caradonna ${ }^{3,5}$, Stacey T. Gray ${ }^{3,6}$, Ahmad R. Sedaghat ${ }^{4}$

' Department of Otolaryngology, Stanford University, Stanford, CA, USA

2 Klinik für Hals-, Nasen-, Ohren- Krankheiten, Hals-und Gesichtschirurgie, Kantonsspital Aarau, Switzerland

${ }^{3}$ Department of Otolaryngology, Harvard Medical School, Boston, MA, USA

${ }^{4}$ Department of Otolaryngology-Head and Neck Surgery, University of Cincinnati College of Medicine, Cincinnati, OH, USA

5 Division of Otolaryngology, Beth Israel Deaconess Medical Center, Boston, MA, USA

Department of Otolaryngology, Massachusetts Eye and Ear Infirmary, Boston, MA, USA
Rhinology 58: 3, 194 - 199, 2020

https://doi.org/10.4193/Rhin19.248

*Received for publication:

July 13, 2019

Accepted: December 9, 2019

\begin{abstract}
Background: The frequencies of systemic antibiotics and oral corticosteroids taken for chronic rhinosinusitis (CRS) indicate poor
\end{abstract} CRS disease control. We sought to determine the validity and responsiveness of these metrics as reflections of CRS disease burden.

Methodology: One hundred and eighty-seven patients undergoing medical management for CRS were recruited. Participants were assessed at two time points: enrollment and a follow-up appointment three to nine months later. At each time point, CRSrelated antibiotic and oral corticosteroid usage in the previous three months was measured, while general and disease-specific quality of life (QOL) was measured using the visual analog scale of the 5-dimension EuroQol questionnaire (EQ-5DVAS) and the 22-item Sinonasal Outcome Test (SNOT-22), respectively.

Results: The frequency of CRS-related antibiotics and oral corticosteroids use was cross-sectionally correlated with EQ-5D VAS and SNOT-22 at the corresponding time points. For participants reporting usage of these medications at enrollment, there was a decrease of 1 course per 3 months for both CRS-related antibiotics and oral corticosteroids. Change in CRS-related antibiotics from enrollment to follow-up was correlated with change in both EQ-5D and SNOT-22 over the same timeframe. The change in CRSrelated oral corticosteroids was correlated with change in both EQ-5D VAS and SNOT-22). These correlations were stronger in the subset of patients who had a change in these metrics over the study period.

Conclusions: The frequencies of CRS-related antibiotic use and oral corticosteroid use are valid and responsive measures of CRS disease burden.

Key words: chronic rhinosinusitis (CRS), antibiotics usage, corticosteroid usage, validation, responsiveness

\section{Introduction}

Chronic rhinosinusitis (CRS) is a common inflammatory disease of the paranasal sinuses ${ }^{(1)}$ that may arise and persist due to heterogeneous etiologies ${ }^{(2-6)}$. CRS is a significant burden on society, leading to billions of dollars in healthcare costs every year as well as a significant quality of life (QOL) detriment for afflicted patients ${ }^{(7-9)}$. CRS decreases $\mathrm{QOL}$ through various disease manifestations including chronic symptomatology ${ }^{(10,11)}$, acute exacerbations of CRS (AECRS) ${ }^{(12)}$, and exacerbations of comorbid pulmonary disease ${ }^{(11,13,14)}$.

Managing the manifestations of CRS to within acceptable limits is the ideal goal of treatment, whether medical or surgical. The degree to which CRS manifestations are within acceptable limits is referred to as disease control. Criteria for CRS disease control were first proposed by the 2012 European Position Paper on Rhinosinusitis and Polyps (EPOS), which recommended assessment of CRS disease elements such as symptom burden, endoscopic burden of disease as well as the use of systemic antibiotics and 
systemic corticosteroids ${ }^{(15)}$. Subsequent studies have highlighted the potential significance of each of these elements of CRS disease control ${ }^{(16-18)}$. The chronic symptomatology of CRS (and its burden on patients) as well as nasal endoscopic burden of CRS have been well characterized ${ }^{(11,19-21)}$. In contrast, systematic antibiotic and systematic corticosteroids usage for CRS as metrics of disease burden are not as well characterized.

The EPOS 2012 criteria describe CRS-related systemic antibiotic usage and systemic corticosteroid usage in the prior 3 months as elements of CRS disease control. Moreover, patient-reported usage of systemic antibiotics and corticosteroids for CRS have been previously described as outcome measures in various studies ${ }^{(22-25)}$. However, the frequency of patient-reported systemic antibiotic or corticosteroid usage has not been fully validated as both valid and responsive measures of CRS disease burden. Given that patient-reported use of CRS-related antibiotics and systemic corticosteroids are increasingly being used in research as outcomes measures of interest after interventions for CRS and also used as an indicator of overall CRS disease control, it is important to establish whether they serve as representative metrics of the CRS disease burden. In this study, we sought to independently show that general and disease-specific QOL measures are reflected by and responsive to patient-reported CRS-related oral antibiotic and oral corticosteroid usage over the preceding three months.

\section{Materials and methods}

Study participants

This study was approved by the Massachusetts Eye and Ear Infirmary Human Studies Committee. We prospectively recruited adult patients of age 18 years or older meeting the American Academy of Otolaryngology - Head and Neck Surgery clinical consensus guideline-established criteria for CRS ${ }^{(26)}$. All study participants provided informed consent for inclusion. Exclusion criteria included comorbid diagnoses of vasculitis, cystic fibrosis, sarcoidosis, immunodeficiency, as well endoscopic sinus surgery within the prior six months.

\section{Study design and data collection}

This was a prospective study of 187 CRS patients undergoing medical management of CRS. All participants were enrolled upon initial presentation to our clinic for CRS. Over the study period, all participants were uniformly treated with intranasal saline irrigations and intranasal corticosteroids, with oral antibiotics or oral corticosteroids given on a patient-by-patient basis, consistent with recommended guidelines for these medications (27). Data was collected at two time points: at enrollment and at follow-up 3 to 9 months later. Any participant who was a current or former tobacco smoker was considered a smoker for this study ${ }^{(28,29)}$. At each time point, all participants completed the 22-item Sinonasal Outcome Test (SNOT-22) as a measure of CRS- specific QOL ${ }^{(30)}$ and the EuroQoL 5-dimensional (EQ-5D VAS) questionnaire from which the visual analog scale (EQ-5D VAS) was used as a measure of general health-related QOL. At each time point, participants were asked to report the number of CRSrelated antibiotics and oral corticosteroids they had used in the past 3 months ${ }^{(29,31)}$. The indications (e.g. AECRS or poor disease control) for CRS-related antibiotics and oral corticosteroids were not recorded. For the purpose of establishing test-retest reliability, a separate set of 15 participants were recruited with follow-up 1 to 3 weeks after the enrollment.

\section{Statistical analysis}

All analyses were performed with the statistical software package R (www.r-project.org). Standard descriptive statistics were performed. All correlations, for example between CRS-related systemic medication usage and measures of QOL, were performed using Spearman correlation. Validation of CRS-related oral antibiotics and oral corticosteroids usage over the previous 3 months was performed by establishing validity, test-retest reliability and responsiveness. Validity was established by showing cross-sectional correlation with SNOT-22 and EQ-5D. Test-retest reliability was assessed by checking for correlation in CRS-related use of oral antibiotics and oral corticosteroids as reported by a set of 15 patients with 1 to 3 weeks between enrollment and follow-up. Responsiveness to medication use was determined by assessing the correlation between change in patient-reported CRS-related oral antibiotics and oral corticosteroids usage and change in SNOT-22 score or change in EQ-5D VAS over the timeframe from enrollment to follow-up.

\section{Results}

\section{Patient characteristics}

A total of 202 participants were included. Of these participants 187 were medically treated for CRS and followed for 3 to 9 months (mean follow-up time 142 days, standard deviation: 49 days). Clinical and demographic characteristics of these participants are summarized in Table 1. At enrollment, 34.2\% of participants reported at least one CRS-related oral antibiotics course in the prior 3 months and $23.0 \%$ of participants reported at least one CRS-related oral corticosteroids course in the prior 3 months. At follow-up, $20.3 \%$ of participants reported least one CRS-related oral antibiotics course and $14.4 \%$ of participants reported at least one CRS-related oral corticosteroids course in the prior 3 months.

\section{Validity of CRS-related oral antibiotic and corticosteroid usage over the prior 3 months}

In order to demonstrate validity, we compared patient-reported CRS-related antibiotics and corticosteroids usage at enrollment to SNOT-22 score and EQ-5D VAS (Figure 1). CRS-related antibiotics usage was correlated with SNOT-22 score $(\rho=0.26, p<0.001)$ 
Table 1. Patient characteristics.

\begin{tabular}{|lc|}
\hline & $\begin{array}{c}\text { Study participants } \\
\text { (N=187) }\end{array}$ \\
\hline $\begin{array}{l}\text { Demographics } \\
\text { Age, mean in years, (SD) }\end{array}$ & $50.9(16.6)$ \\
\hline $\begin{array}{l}\text { Gender } \\
\text { Male }\end{array}$ & $49.7 \%$ \\
\hline Female & $50.3 \%$ \\
\hline Smoking & $37.4 \%$ \\
\hline Comorbidities & \\
\hline Aeroallergen hypersensitivity & $48.7 \%$ \\
\hline Asthma & $32.1 \%$ \\
\hline Aspirin sensitivity & $4.3 \%$ \\
\hline $\begin{array}{l}\text { CRS characteristics at enrollment } \\
\text { Nasal polyps }\end{array}$ & $45.5 \%$ \\
\hline Previous sinus surgery & $35.8 \%$ \\
\hline Intranasal saline use & $24.1 \%$ \\
\hline Intranasal corticosteroid use & $43.9 \%$ \\
\hline Patient-reported outcome measures at & \\
\hline enrollment & \\
\hline SNOT-22 score, mean (SD) & $36.9(21.1)$ \\
\hline $\begin{array}{l}\text { Number of CRS-related antibiotics taken in } \\
\text { last } 3 \text { months, mean (SD) }\end{array}$ & $0.5(0.8)$ \\
\hline $\begin{array}{l}\text { Number of CRS-related oral corticosteroids } \\
\text { taken in last } 3 \text { months, mean (SD) }\end{array}$ & $0.3(0.6)$ \\
\hline $\begin{array}{l}\text { SNOT-22 score, mean (SD) } \\
\text { last } 3 \text { months, mean (SD) }\end{array}$ \\
\hline $\begin{array}{l}\text { Number of CRS-related oral corticosteroids } \\
\text { taken in last } 3 \text { months, mean (SD) }\end{array}$ \\
\hline
\end{tabular}

SD: Standard deviation

and EQ-5D VAS ( $\rho=-0.23, p=0.002)$. Similarly, at enrollment CRSrelated corticosteroids usage was correlated with SNOT-22 score ( $\rho=0.24, p=0.001)$ and EQ-5D VAS $(\rho=-0.20, p=0.008)$.

Test-retest reliability of CRS-related antibiotic and corticosteroid usage over the prior 3 months

A set of 15 participants reported their CRS-related antibiotics and oral corticosteroids usage over the prior 3 months at time points 1 to 3 weeks apart. Test-retest reliability was assessed by checking for correlation between participants' responses between the two times points. For this set of 15 participants, the reported CRS-related antibiotics usage over the prior 3 months was significantly correlated $(\rho=0.85, p<0.001)$ over the two time points. Also, the reported CRS-related oral corticosteroids usage over the prior 3 months was significantly correlated $(\rho=0.87$, $\mathrm{p}<0.001)$.
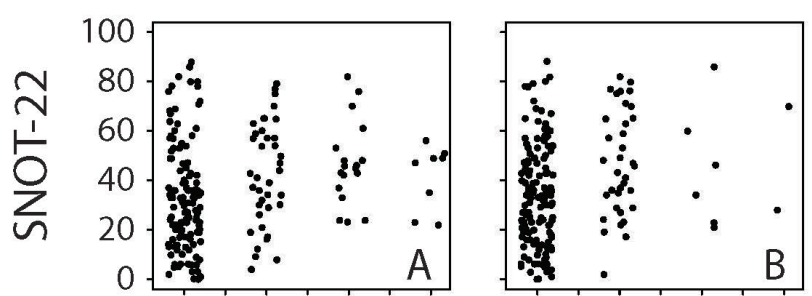

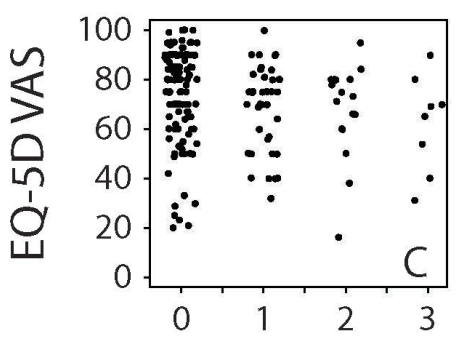

Antibiotics

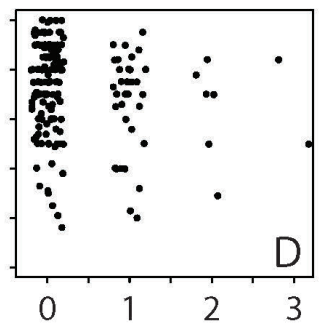

Corticosteroids
Figure 1. Scatterplots of SNOT-22 score vs. A) reported CRS-related oral antibiotics courses in the prior 3 months and $B$ ) reported CRS-related oral corticosteroids courses in the prior 3 months. Scatterplots of EQ-5D vs. C) reported CRS-related oral antibiotics courses in the prior 3 months and D) reported CRS-related corticosteroids courses in the prior 3 months.

Responsiveness of CRS-related antibiotic and corticosteroid usage over the prior 3 months

Responsiveness was established by assessing the change in reported CRS-related antibiotics and oral corticosteroids usage over the prior 3 months from enrollment to the follow-up time point after the participants had been medically managed for CRS. Over the follow-up period, there was a mean decrease in CRS-related antibiotics usage by 0.3 courses per 3 months and a mean decrease in CRS-related oral corticosteroids usage by 0.1 courses per 3 months. Examining the subset of participants who reported at least 1 course of CRS-related antibiotics in the 3 months prior to enrollment, the mean decrease in CRS-related oral antibiotics usage was 1.2 courses per 3 months over the study period. Likewise focusing on the subset of participants who reported at least 1 course of CRS-related oral corticosteroids in the 3 months prior to enrollment, the mean decrease in CRS-related oral corticosteroids usage was 0.9 courses per 3 months over the study period. Thus with appropriate medical management, the usage of these systemic medications for CRS significantly decreased.

Responsiveness was also established by assessing the correlation between changes in patient-reported CRS-related antibiotics and oral corticosteroids usage over the prior 3 months, and changes in SNOT-22 and in EQ-5D VAS over the 3 to 9 month follow-up period (Figure 2). Over the follow-up period, the change in CRS-related antibiotics usage was correlated with change in SNOT-22 score $(\rho=0.17, p=0.020)$ and change in EQ- 

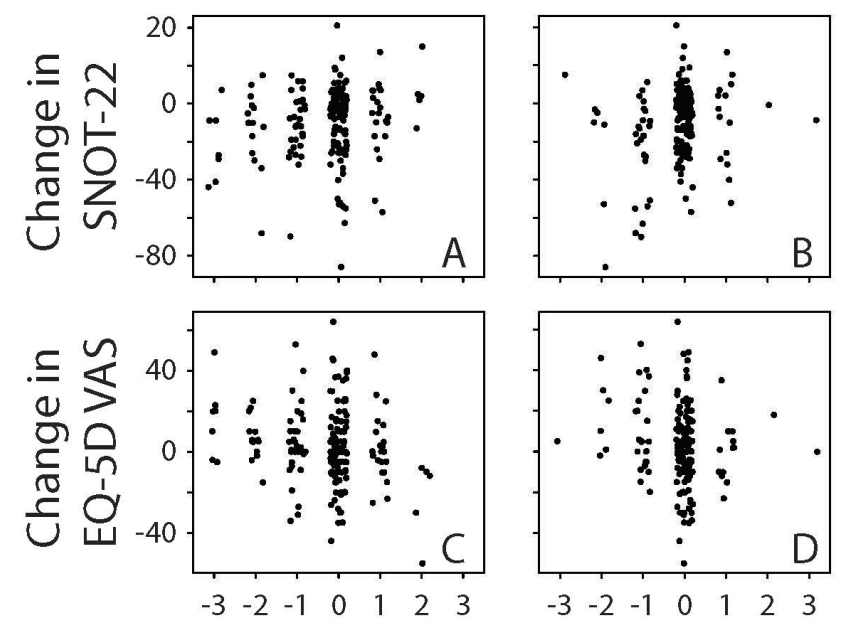

\section{Change in antibiotics}

CRS and also revealed the use of antibiotics and oral corticosteroids for CRS is a distinct aspect of the CRS disease course ${ }^{(12,34-36)}$. However, patient-reported, CRS-related antibiotics and oral corticosteroids usage has not been validated as a metric for CRS disease burden. In this study, we seek to validate patientreported, CRS-related antibiotics and oral corticosteroids usage as valid, reliable and responsive measures of disease burden. Previous work has shown that use of antibiotics and oral corticosteroids for CRS is directly associated with reduced $\mathrm{QOL}$ as well as productivity ${ }^{(11,12)}$. The association between the use of these systemic medications and downstream consequences (QOL and productivity) of CRS are independent of other disease aspects, such as symptom burden. For example, the use of CRS-related antibiotics and oral corticosteroids are independently associated with pulmonary exacerbations in asthmatic CRS patients ${ }^{(37)}$ and CRS-related productivity in asthmatic CRS patients (11). As important elements of the CRS disease course and CRS disease control, validation of CRS-related usage of antibiotics and oral corticosteroids as outcome measured is needed.

In this study, we show that patient-reported, CRS-related antibiotics and oral corticosteroids usages in the prior 3 months are valid, reliable and responsive measures of CRS disease burden. We showed criterion validity by showing that the use of these systemic medications was correlated with general health-related and disease-specific QOL. We showed responsiveness in two ways. First, we show that after the participants used appropriate medical management for their CRS, there was a mean decrease in the usage of CRS-related antibiotics and oral corticosteroids. Of note, when we focused on the subset of participants who had reported at least some usage of these systemic medications at enrollment, appropriate medical management of CRS was associated with a mean decrease of approximately 1 course of each medication per 3 month period. We also showed responsiveness of patient-reported CRS-related antibiotics and CRS-related oral corticosteroids usage in the prior 3 months by showing that the changes in these quantities correlated with changes in general health-related and disease-specific QOL over the study period. Moreover, we find the test-retest reliability of these metrics are quite high. We feel that these results provide credence for the use of these metrics to study and research CRS clinical outcomes. Moreover, the concept of CRS disease control is increasingly being proposed as a unifying measure that synthesizes multiple CRS disease manifestations upon which to base CRS treatment decisions. CRS-related systemic antibiotic and corticosteroid usage in the prior 3 months has been previously recommended as a key component of CRS disease control (15). Subsequent work has shown that the use of these medications is consistently considered by physicians as a reflection of poor CRS control ${ }^{(18,40)}$. Our results here also provide support for the use of patient-reported, CRS-related oral antibiotics and oral corticosteroids usages as valid and reliable metrics that can be 
used in the clinical setting.

Our results open the door for future study of these metrics to understand the indications for which CRS-related antibiotics and oral corticosteroids are taken. It is very possible that the use of these medications for the resolution of discrete AECRS may be reflective of a different level of disease burden than in the patient whose disease is so severe that these medications are required for suppression of baseline symptomatology, although in both cases patients may be exposed to similar levels of medication-related side effects. This is an exciting area of investigation that will require not only systematic investigation of patient outcomes in each scenario but also consensus building around value judgements related to systemic medication usage as a reflection of poor control due to the underlying indication or the risk of harm (i.e., side effects) to the patient.

Our study should be interpreted in the context of its limitations. Although we show that patient-reported CRS-related usage of oral antibiotics and oral corticosteroids in the past 3 months is reliable, this nevertheless requires patients to recall their usage of these medications which may be influenced by recall bias. The recall period of the SNOT-22 (2 weeks), the EQ-5D VAS (1 day) and CRS-related antibiotics and oral corticosteroids in the past 3 months are all different. However, that we nevertheless find significant correlations speaks to the strength of the relationships between these CRS outcomes. Additionally, the antibiotics and corticosteroids were prescribed by multiple providers, which is a pragmatic aspect of our study design since CRS patients may seek care from any number of physicians. Furthermore, even though general guidelines exist for the use of these medications the prescription of oral antibiotics and oral corticosteroids for CRS is physician-dependent to some extent. Moreover, the negative impact of oral antibiotics and corticosteroids usage goes beyond poor CRS control but is also reflective of side effects and adverse events that patients may experience from these medications ${ }^{(41,42)}$.

\section{Conclusion}

Patient-reported CRS-related antibiotics and corticosteroids usage are valid and responsive measures of disease burden. CRS disease burden that is reflected by CRS-related antibiotics and oral corticosteroids usage may not only be indicative of poor CRS disease control (e.g. recurrent AECRS or high disease severity) but may also reflect side effects of these systemic medications.

\section{Acknowledgements}

The authors gratefully acknowledge the contribution of the patients who participated in this study.

No funding was used to support this study.

\section{Authorship contribution}

KMP designed and performed the study, and wrote/revised the manuscript; MMS, ETS, RT, DSC, STG performed the study and wrote/revised the manuscript; ARS conceived, designed, and performed the study and wrote/revised the manuscript.

\section{Conflict of interest}

The authors declare no conflicts of interest.

\section{References}

1. Hastan D, Fokkens WJ, Bachert Cet al. Chronic rhinosinusitis in Europe--an underestimated disease. A GA(2)LEN study. Allergy 2011; 66:1216-1223.

2. Barshak MB, Durand ML. The role of infection and antibiotics in adult chronic rhinosinusitis. Laryngoscope Investig Otolaryngol. 2017 Jan 23;2(1):36-42.

3. Marcus S, Roland LT, DelGaudio JM, Wise SK. The relationship between allergy and chronic rhinosinusitis. Laryngoscope Investig Otolaryngol. 2018 Dec 20;4(1):1317.

4. Sedaghat AR, Gray ST, Wilke CO, Caradonna DS. Risk factors for development of chronic rhinosinusitis in patients with allergic rhinitis. Int Forum Allergy Rhinol 2012; 2:370375.

5. Sedaghat AR, Phipatanakul W, Cunningham MJ. Atopy and the development of chronic rhinosinusitis in children with allergic rhinitis. J Allergy Clin Immunol Pract 2013; 1:689691 e681-682.

6. Stevens WW, Peters AT. Immunodeficiency in chronic sinusitis: recognition and treat- ment. Am J Rhinol Allergy 2015; 29:115-118.

7. Caulley L, Thavorn K, Rudmik L, Cameron C, Kilty SJ. Direct costs of adult chronic rhinosinusitis by using 4 methods of estimation: Results of the US Medical Expenditure Panel Survey. J Allergy Clin Immunol 2015; 136:1517-1522.

8. Rudmik L. Economics of Chronic Rhinosinusitis. Curr Allergy Asthma Rep. 2017;17(4):20

9. DeConde AS, Soler ZM. Chronic rhinosinusitis: Epidemiology and burden of disease. Am J Rhinol Allergy 2016; 30:134-139.

10. Hoehle LP, Phillips KM, Bergmark RW, Caradonna DS, Gray ST, Sedaghat AR. Symptoms of chronic rhinosinusitis differentially impact general health-related quality of life. Rhinology 2016; 54:316-322.

11. Speth MM, Hoehle LP, Phillips KM, Caradonna DS, Gray ST, Sedaghat AR. Changes in chronic rhinosinusitis symptoms differentially associate with improvement in general health-related quality of life. Ann Allergy Asthma Immunol 2018.

12. Phillips KM, Hoehle LP, Bergmark RW, Caradonna DS, Gray ST, Sedaghat AR.
Acute Exacerbations Mediate Quality of Life Impairment in Chronic Rhinosinusitis. J Allergy Clin Immunol Pract 2017; 5:422-426.

13. Phillips KM, Hoehle LP, Bergmark RW et al Chronic rhinosinusitis severity is associated with need for asthma-related systemic corticosteroids. Rhinology 2017; 55:211-217.

14. Phillips KM, Hoehle LP, Caradonna DS, Gray ST, Sedaghat AR. Association of severity of chronic rhinosinusitis with degree of comorbid asthma control. Ann Allergy Asthma Immunol 2016; 117:651-654.

15. Fokkens WJ, Lund VJ, Mullol Jet al. European Position Paper on Rhinosinusitis and Nasal Polyps 2012. RhinologySupplement 2012; (23):1-298.

16. Snidvongs K, Heller GZ, Sacks R, Harvey RJ. Validity of European position paper on rhinosinusitis disease control assessment and modifications in chronic rhinosinusitis. Otolaryngol Head Neck Surg. 2014 Mar;150(3):479-86.

17. Banglawala SM, Schlosser RJ, Morella Ket al. Qualitative development of the sinus control test: a survey evaluating sinus symptom control. Int Forum Allergy Rhinol. 2016; 
6:491-499.

18. Sedaghat AR, Hoehle LP, Gray ST. Chronic rhinosinusitis control from the patient and physician perspectives. Laryngoscope Investig Otolaryngol 2018; 3:419-433.

19. Feng $A L$, Wesely $N C$, Hoehle LPet al. A validated model for the 22-item Sino-Nasa Outcome Test subdomain structure in chronic rhinosinusitis. Int Forum Allergy Rhinol 2017; 7:1140-1148.

20. Psaltis AJ, Li G, Vaezeafshar R, Cho KS, Hwang PH. Modification of the LundKennedy endoscopic scoring system improves its reliability and correlation with patient-reported outcome measures. Laryngoscope 2014; 124:2216-2223.

21. Snidvongs K, Dalgorf D, Kalish L, Sacks R, Pratt E, Harvey RJ. Modified Lund Mackay Postoperative Endoscopy Score for defining inflammatory burden in chronic rhinosinusitis. Rhinology 2014; 52:53-59.

22. Bhandarkar ND, Mace JC, Smith TL. Endoscopic sinus surgery reduces antibiotic utilization in rhinosinusitis. Int Forum Allergy Rhinol. 2011; 1:18-22.

23. Yamasaki A, Hoehle LP, Phillips KMet al. Association between systemic antibiotic and corticosteroid use for chronic rhinosinusitis and quality of life. Laryngoscope 2018; 128:37-42.

24. Gliklich RE, Metson R. Techniques for outcomes research in chronic sinusitis. Laryngoscope 1995; 105:387-390.

25. Ramakrishnan VR, Mace JC, Soler ZM, Smith TL. Is greater antibiotic therapy prior to ESS associated with differences in surgical outcomes in CRS? Laryngoscope 2019; 129:558-566

26. Rosenfeld RM, Piccirillo JF, Chandrasekhar SS et al. Clinical practice guideline (update) adult sinusitis. Otolaryngol Head Neck Surg. 2015: 152:S39.

27. Orlandi RR, Kingdom TT, Hwang PHet al. International Consensus Statement on Allergy and Rhinology: Rhinosinusitis. Int Forum Allergy Rhinol. 2016; 6 Suppl 1:S209.

28. Hoehle LP, Phillips KM, Caradonna DS, Gray
ST, Sedaghat AR. A contemporary analysis of clinical and demographic factors of chronic rhinosinusitis patients and their association with disease severity. Irish J Med Sci. 2017

29. Phillips KM, Hoehle L, Bergmark RW Caradonna DS, Gray ST, Sedaghat AR. Reversal of Smoking Effects on Chronic Rhinosinusitis after Smoking Cessation. Otolaryngol Head Neck Surg 2017; 157:737742.

30. Hopkins C, Gillett S, Slack R, Lund VJ, Browne JP. Psychometric validity of the 22-item Sinonasal Outcome Test. Clin Otolaryngol. 2009; 34:447-454.

31. Speth MM, Gaudin RA, Hoehle LPet al. Reciprocal Predictive Accuracy of Sinonasal Symptom Severity, Nasal Endoscopy, and Frequency of Past Chronic Rhinosinusitis Exacerbations. Otolaryngol Head Neck Surg 2018; 159:766-773.

32. EPR-3. NAEPP expert panel report 3: guidelines for the diagnosis and treatment of asthma. Bethesda (MD): US Department of Health and Human Services; National Institutes of Health; National Heart, Lung, and Blood Institute, 2007.

33. Reddel HK, Bateman ED, Becker A, et al. A summary of the new GINA strategy: a roadmap to asthma control. Eur Respir J. 2015 46:622-639.

34. Gray ST, Phillips KM, Hoehle LPet al Utilization patterns of systemic corticosteroid use for chronic rhinosinusitis. Acta Otolaryngol 2018; 138:153-158.

35. Ramakrishnan VR, Mace JC, Soler ZM, Smith TL. Examination of high-antibiotic users in a multi-institutional cohort of chronic rhinosinusitis patients. Int Forum Allergy Rhinol. 2017

36. Ramakrishnan VR, Mace JC, Soler ZM, Smith TL. Is greater antibiotic therapy prior to ESS associated with differences in surgical outcomes in CRS? Laryngoscope. 2019 Mar;129(3):558-566.

37. Banoub RG, Phillips KM, Hoehle LP, Caradonna DS, Gray ST, Sedaghat AR.
Relationship between chronic rhinosinusitis exacerbation frequency and asthma control. Laryngoscope 2018; 128:1033-1038.

38. Divekar RD, Samant S, Rank MA, et al. Immunological profiling in chronic rhinosinusitis with nasal polyps reveals distinct VEGF and GM-CSF signatures during symptomatic exacerbations. Clin Exp Allergy. 2015; 45:767-778.

39. Rank MA, Wollan P, Kita H, Yawn BP. Acute exacerbations of chronic rhinosinusitis occur in a distinct seasonal pattern. J Allergy Clin Immunol. 2010; 126:168-169.

40. Kohli P, Soler ZM, Storck KA, Shahangian A, Banglawala SM, Schlosser RJ. Responsiveness and reliability of the Sinus Control Test in chronic rhinosinusitis. Rhinology 2017; 55:39-44.

41. Head K, Chong LY, Piromchai P, et al. Systemic and topical antibiotics for chronic rhinosinusitis. Cochrane Database Syst Rev. 2016; 4:CD011994.

42. 42. Poetker DM, Smith TL. What rhinologists and allergists should know about the medico-legal implications of corticosteroid use: a review of the literature. Int Forum Allergy Rhinol. 2012; 2:95-103.

Katie M. Phillips, MD

Department of Otolaryngology

Stanford University

801 Welch Rd

Stanford, CA 94305

USA

Tel: +001 650-725-6500

E-mail:kphillipsm@gmail.com 